\title{
Digital Microfluidic Biochips: A Vision for Functional Diversity and More than Moore
}

\author{
Krishnendu Chakrabarty \\ Department of Electrical and Computer Engineering \\ Duke University. Durham, NC 27708, USA
}

\begin{abstract}
Microfluidics-based biochips are revolutionizing highthroughput sequencing, parallel immunoassays, clinical diagnostics, and drug discovery. These devices enable the precise control of nanoliter volumes of biochemical samples and reagents. Compared to conventional laboratory procedures, which are cumbersome and expensive, miniaturized biochips offer the advantages of higher sensitivity, lower cost due to smaller sample and reagent volumes, system integration, and less likelihood of human error. This embedded tutorial paper provides an overview of droplet-based "digital" microfluidic biochips. It describes emerging computer-aided design (CAD) tools for the automated synthesis and optimization of biochips from bioassay protocols. Recent advances in fluidic-operation scheduling, module placement, droplet routing, pin-constrained chip design, and testing are presented.
\end{abstract}

\section{INTRODUCTION}

Advances in digital microfluidics have led to the promise of miniaturized biochips for applications such as immunoassays for point-of-care medical diagnostics, DNA sequencing, and the detection of airborne particulate matter [1-4]. These devices enable the precise control of nanoliter droplets of biochemical samples and reagents, and integrated circuit (IC) technology can be used to transport and process "biochemical payload" in the form of tiny droplets. Biochips facilitate the convergence of electronics with the life sciences, and they integrate on-chip various bioassay operations, such as sample preparation, analysis, separation, and detection [2]. Compared to conventional laboratory procedures, which are cumbersome and expensive, miniaturized biochips offer the advantages of higher sensitivity, lower cost due to smaller sample and reagent volumes, system integration, and less likelihood of human error. As a result, non-traditional biomedical applications and markets are opening up fundamentally new uses for ICs.

However, continued growth in this emerging field depends on advances in chip/system integration. In particular, design methods are needed to ensure that biochips are as versatile as the macro-labs that they are intended to replace. The few commercial biochips available today (e.g., from Agilent, Fluidigm, Caliper, I-Stat, BioSite, etc.) are specific to an application and they offer no flexibility to the user. Intel recently announced the Health Guide PHS6000 product for home patients, but the underlying technology does not exploit the benefits of reconfigurable microfluidics.

This embedded tutorial paper is focused on droplet-based "digital" microfluidic biochips. The digital microfluidics platform offers the flexibility of dynamic reconfigurability and software-based control of multifunctional biochips. Next the paper describes emerging computer-aided design (CAD) tools for the automated synthesis and optimization of biochips from bioassay protocols. Recent advances on fluidic-operation scheduling, module placement, droplet routing, testing, and dynamic reconfiguration are also presented. These techniques allow biochip users to concentrate on the development of nanoscale bioassays, leaving chip optimization and implementation details to design-automation tools.

It is expected that an automated design flow will transform biochip research and use, in the same way as design automation revolutionized IC design in the 80 s and 90s. This approach is especially aligned with the vision of functional diversification and "More than Moore", as articulated in the ITRS 2007, which highlights "Medical" as being a "System Driver" for the future [6]. Users will adapt more easily to emerging technology if appropriate design methods/tools and in-system automation methods are available.

The rest of this paper is organized as follows. Section II describes biochip technology platforms, including digital microfluidics. Section III presents synthesis techniques, including solutions published in the literature for operation scheduling, module placement, and droplet routing. Section IV describes pin-constrained chip methods. Section V presents advances in testing, including fault models and fault detection. Finally, Section VI concludes the paper.

\section{TECHNOLOGY PLATFORMS}

Early biochips were based on the concept of a DNA microarray, which is a piece of glass, plastic or silicon substrate on which pieces of DNA, i.e., probes, have been affixed. There are a number of commercial microarrays available in the marketplace today, e.g., GeneChip ${ }^{\circledR}$ DNAarray from Affymetrix, NanoChip ${ }^{\circledR}$ microarray from Nanogen, and DNA microarray from Agilent. A drawback of these arrays is that they are "passive chips"; they are neither reconfigurable nor can they be used for sample preparation.

The basic idea of a microfluidic biochip is to integrate all necessary functions for biochemical analysis using microfluidics technology. These micro-total-analysis-systems are more versatile than microarrays. Integrated functions include assay operations, detection, and sample preparation.

\section{A. Continuous-Flow Microfluidics}

Traditional (continuous-flow) microfluidic technologies are based on the continuous flow of liquid through microfabricated channels [5, 7-8]. Continuous-flow systems are 
inherently difficult to integrate because the parameters that govern flow field (e.g. pressure, fluid resistance, electric field strength) vary along the flow-path, making the flow at any location dependent upon the properties of the entire system. Moreover, unavoidable shear flow and diffusion in microchannels make it difficult to eliminate intersample contamination and dead volumes. Furthermore, since structure and functionality are so tightly coupled, each system is only appropriate for a narrow class of applications.

\section{B. Digital Microfluidics}

A digital microfluidic biochip utilizes electrowetting on dielectric (EWOD) to manipulate and move microliter or nanoliter droplets containing biological samples on a twodimensional electrode array [2, 9-12]. A unit cell in the array includes a pair of electrodes that acts as two parallel plates. The bottom plate contains a patterned array of individually controlled electrodes, and the top plate is coated with a continuous ground electrode. A droplet rests on a hydrophobic surface over an electrode. It is moved by applying a control voltage to an electrode adjacent to the droplet and, at the same time, deactivating the electrode just under the droplet. Using interfacial tension gradients, droplets can be moved to any location on a two-dimensional array.

The division of a volume of fluid into discrete, independently controllable "packets" or droplets, provides several advantages over continuous-flow. The reduction of microfluidics to a set of basic repeated operations (i.e., "move one unit of fluid one distance unit") enables a cell-based design approach. By varying the patterns of control-voltage activation, fluid-handling operations such as droplet merging, splitting, mixing, and dispensing can be easily executed. The platform offers dynamic reconfigurability, since fluidic operations can be performed anywhere on the array. Droplet routes and operation scheduling results are programmed into a microcontroller that drives electrodes in the array.

To address the need for low-cost, PCB technology has been employed for inexpensive fabrication. Using a copper layer for the electrodes, solder mask as the insulator, and a Teflon AF coating for hydrophobicity, the microfluidic array can be fabricated using an existing PCB process.

\section{SYNTHESIS METHODS}

In this section, we examine a progression of CAD problems related to biochip synthesis.

\section{A. Scheduling and Module Placement}

Recent years have seen growing interest in the automated design and synthesis of microfluidic biochips [13, 19, 21, 2529]. Optimization goals here include the minimization of assay completion time, minimization of chip area, and higher defect tolerance. The minimization of the assay completion time is essential for environmental monitoring applications where sensors can provide early warning. Real-time response is also necessary for surgery and clinical diagnostics. Finally, biological samples are sensitive to the environment and to temperature variations, and it is difficult to maintain an optimal clinical or laboratory environment on chip.
One of the first published methods for biochip synthesis decoupled high-level synthesis from physical design [13]. Architectural-level synthesis for microfluidic biochips can be viewed as the problem of scheduling assay functions and binding them to a given number of resources so as to maximize parallelism, thereby decreasing response time. A behavioral model for a set of bioassays is first obtained from their laboratory protocols. Architectural-level synthesis is then used to generate a macroscopic structure of the biochip. Geometry-level synthesis (physical design) addresses the placement of resources and the routing of droplets to satisfy objectives such as area or throughput. It creates the final layout of the biochip, consisting of the placement of microfluidic modules such as mixers and storage units, the routes that droplets take between different modules, and other geometrical details [21].

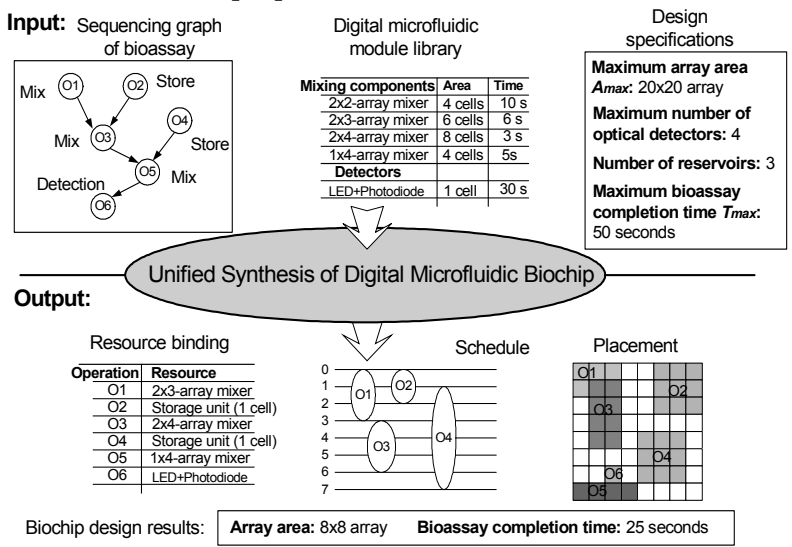

Fig. 1. An example illustrating system-level synthesis [16].

A key problem in the geometry-level synthesis of biochips is the placement of microfluidic modules such as different types of mixers and storage units. Since digital microfluidicsbased biochips enable dynamic reconfiguration of the microfluidic array during run-time, they allow the placement of different modules on the same location during different time intervals. A simulated annealing-based heuristic approach has been developed to solve the NP-complete problem in a computationally efficient manner [21].

Architectural synthesis is based on rough estimates for placement costs such as the area of the microfluidic modules. These estimates provide lower bounds on the exact biochip area, since the overheads due to spare cells and cells used for droplet transportation are not known a priori. However, it cannot be accurately predicted if the biochip design meets system specifications, e.g., maximum allowable array area and upper limits on assay completion times, until both high-level synthesis and physical design are carried out. [16] proposed a unified system-level synthesis method for microfluidic biochips based on parallel recombinative simulated annealing (PRSA), which offers a link between these two steps.

The design flow is illustrated in Fig. 1. First, the different bioassay operations (e.g. mixing and dilution), and their mutual dependences are represented using a sequencing graph. Next, a combination of simulated annealing and genetic 
algorithms are used for unified resource binding, operation scheduling, and module placement. A chromosome is used to represent each candidate solution, i.e., a design point. In each chromosome, operations are randomly bound to resources. Based on the binding results, list scheduling is used to determine the start times of operations, i.e., each operation starts with a random latency after its scheduled time. Finally, a module placement is derived based on the resource binding and the schedule of fluidic operations. A weighted sum of area- and time-cost is used to evaluate the quality of the design. The design is improved through a series of genetic evolutions based on PRSA. It generates an optimized schedule of bioassay operations, the binding of assay operations to resources, and a layout of the microfluidic biochip.

Efficient reconfiguration techniques have been developed to bypass faulty unit cells in the microfluidic array. A microfluidic module containing a faulty unit cell can easily be relocated to another part of the microfluidic array by changing the control voltages applied to the corresponding electrodes. Defect tolerance can also be achieved by including redundant elements in the system; these elements can be used to replace faulty elements through reconfiguration techniques [17]. Another method is based on graceful degradation, in which all elements in the system are treated in a uniform manner, and no element is designated as a spare [18].

The top-down synthesis flow described above unifies architecture level design with physical-level module placement. However, it suffers from two drawbacks. For operation scheduling, it is assumed that the time cost for droplet routing is negligible, which implies that droplet routing has no influence on the operation completion time. While generating physical layouts, the synthesis tool in [16] provides only the layouts of the modules and it leaves droplet routing pathways unspecified. The assumption of negligible droplet transportation times is valid for small microfluidic arrays. However, for large arrays and for biochemical protocols that require several concurrent fluidic operations onchip, the droplet transportation time is significant and routing complexity is non-trivial. This problem is addressed in the next subsection.

\section{B. Droplet Routing}

A key problem in biochip physical design is droplet routing between modules, and between modules and I/O ports (i.e., on-chip reservoirs). The dynamic reconfigurability inherent in digital microfluidics allows different droplet routes to share cells on the microfluidic array during different time intervals. In this sense, the routes in microfluidic biochips can be viewed as virtual routes, which make droplet routing different from the classical wire VLSI routing problem.

The first method for droplet routing in biochips was published in [19]. The main objective in routing is to find droplet routes with minimum lengths, where route length is measured by the number of cells in the path from the starting point to the destination.

During droplet routing, a minimum spacing between droplets must be maintained to prevent accidental mixing, except for the case when droplet merging is desired (e.g., in 3- pin nets). Fluidic constraint rules in [19] need to be satisfied in order to avoid undesirable mixing. The microfluidic modules placed on the array are viewed as obstacles in droplet routing. In order to avoid conflicts between droplet routes and assay operations, a segregation region is added to wrap around the functional region of microfluidic modules. Another constraint in droplet routing is given by an upper limit on droplet transportation time. The delay for each droplet route should not exceed some maximum, e.g., $10 \%$ of a time-slot used in scheduling, in order that the droplet-routing time can be ignored for scheduling assay operations [16].

Since a digital microfluidic array can be reconfigured dynamically at run-time, a series of 2-D placement configurations of modules in different time spans are obtained in the module placement phase [48]. Therefore, the droplet routing is decomposed into a series of sub-problems. A complete droplet-routing solution is obtained by solving these sub-problems sequentially.

Based on this problem formulation, a two-stage routing method has been proposed in [21]. In the first stage, $M$ alternative routes for each net are generated. In the second stage, a single route from the $M$ alternatives for each net is selected independent of the routing order of nets. This method also exploits the features of dynamic reconfigurability and independent controllability of electrodes to modify droplet pathways to override potential violation of fluidic constraints.

Droplet routing should be considered in the synthesis flow for digital microfluidics, in order to generate a routable synthesized design for the availability of routing paths. [25] proposed a method to incorporate droplet-routability in the PRSA-based synthesis flow. This method estimates the droplet-routability using two metrics. It adopts the average module distance (over all interdependent modules) as the first design metric to guarantee the routability of modules in the synthesized biochip. It also adopts the maximum module distance as the second design metric to approximate the maximum length of droplet manipulation. Since synthesis results with high routability values are more likely to lead to simple and efficient droplet pathways, this method incorporates the above two metrics into the fitness function by a factor that can be fine-tuned according to different design specifications to control the PRSA-based procedure. For each chromosome considered in the PRSA-based synthesis flow, this method calculates both the average and maximum module distance. Candidate designs with low routability are discarded during evolution. Thus, the synthesis procedure guarantees that the routing complexity is reduced for the synthesized biochip, while meeting constraints on array size and bioassay processing time.

We ran the defect-tolerant routing-aware and defectoblivious routing-aware algorithms under a set of combinations of weights in the fitness function for a protein assay example. We carried out random defect injection into each design and obtain its failure rate. We mapped each design $G$ to a $3 \mathrm{D}$ point $\left(T_{G}, A_{G}, F_{G}\right)$, where $T_{G}, A_{G}, F_{G}$ are completion time, chip area, and failure rate of the design, respectively. A point $\left(T_{G}, A_{G}, F_{G}\right)$ is referred to as a feasibility boundary point 


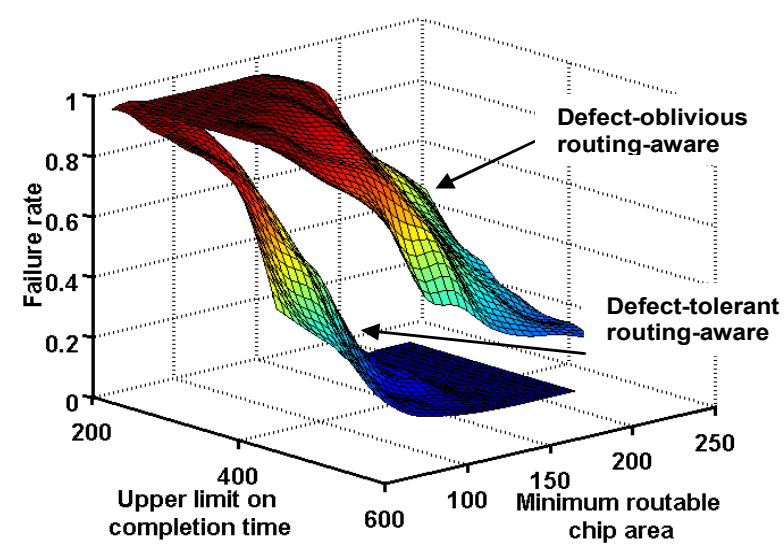

Fig. 2. Feasibility frontier surface and feasible design region for defecttolerant and defect-oblivious routing-aware synthesis methods.

if there are no other points $\left(T_{m}, A_{m}, F_{m}\right)$ such that $T_{m}<T_{G}, A_{m}$ $<A_{G}$, and $F_{m}<F_{G}$. A feasibility frontier surface is obtained by connecting all the feasibility boundary points, as shown in Fig. 2. The feasible design region corresponds to the space above the feasible surface. Any design specification can be met whose corresponding is point located in this region; otherwise, no feasible design exists for this specification. As shown in Fig. 2, defect-tolerant routing-aware synthesis leads to a lower-feasibility frontier surface and a larger feasible design space as compared to the defect-oblivious method.

\section{PIN-CONSTRAINED CHIP DESIGN}

Electrode addressing is an important problem in biochip design. It refers to the manner in which electrodes are connected to and controlled by input pins. Early designautomation techniques relied on the availability of a directaddressing scheme. For large arrays, direct-addressing schemes lead to a large number of control pins, and the associated interconnect routing problem significantly adds to the product cost. In this section, we describe a number of pinconstrained biochip design methods.

\section{A. Droplet-Trace-Based Array Partitioning}

An array-partitioning-based pin-constrained design method of digital microfluidic biochips proposed in [22]. This method uses array partitioning and careful pin assignment to reduce the number of control pins. The key idea is to "virtually" partition the array into regions. The partitioning criterion here is to ensure at most one droplet is included in each partition. The droplet trace, defined as the set of cells traversed by a single droplet, serves as the basis for generating the array partitions. The droplet trace can be easily extracted from the droplet routing information and the placement of the modules to which it is routed. If droplets traces intersect on the array, the partitions derived by this method overlap in some regions. Sets of pins from an "overlapping" partition cannot be used in the overlapped region since the reuse of the pins may lead to droplet interference. The solution to this problem is to make the overlapping region a new partition, referred to as the overlapping partition, and use direct addressing (one-to-one mapping) for it. An efficient algorithm for mapping control pins to the electrodes in a partition has also been developed.

The above approach can be integrated into the droplet-tracebased array partitioning method to generate dropletinterference-free layouts with a minimum number of pins. However, this method requires detailed information about the scheduling of assay operations, microfluidic module placement, and droplet routing pathways. Thus, the array design in such cases is specific to a target biofluidic application.

\section{B. Cross-Referencing-Based Droplet Manipulation}

An alternative design method based on a cross-reference driving scheme is presented in $[26,30]$. This method allows control of an $N \times M$ grid array with only $N+M$ control pins. The electrode rows are patterned on both the top and bottom plates, and placed orthogonally. However, due to electrode interference, this design cannot handle the simultaneous movement of more than two droplets. For the concurrent manipulation of multiple droplets on a cross-referencing-based biochip, multiple row and column pins must be selected to activate the destination cells, i.e., cells to which the droplets are supposed to move. However, the selected row and column pins may also result in the activation of cells other than the intended droplet destinations.

A solution based on destination-cell categorization has been proposed to tackle the above problem. The key idea is to group the droplet movements according to their destination cells. A group consists of droplets whose destination cells share the same column or row. In this way, the manipulation of multiple droplets is ordered in time; droplets in the same group can be moved simultaneously without electrode interference, but the movements for the different groups must be sequential. The problem of finding the minimum number of groups can be directly mapped to the problem of determining a minimal clique partition from graph theory. A linear-time heuristic algorithm based on row-scanning and column-scanning has been used to derive the clique partitions.

\section{Broadcast-Addressing Method}

A broadcast-addressing based design technique for pinconstrained and multi-functional biochips has been developed in [27]. To execute a specific bioassay, routing and scheduling information must be stored in the form of electrode activation sequences, where each bit representing the status of the electrode at a specific time-step. The status can be either " 1 " (activate), " 0 " (deactivate) or " $F$ " (floating). The "floating" status is represented using the symbol " $\mathrm{x}$ " and refer to it as "don't-care". Each electrode activation sequence contains several don't-care terms, which can be replaced by " 1 " or " 0 ". If two sequences can be made identical by careful replacing these don't-care terms with " 0 " or " 1 ", they are referred to as compatible sequences. Compatible sequences can be generated from a single signal source. 
TABLE I

EXAMPLES OF FAULT MODELS FOR DIGITAL MICROFLUIDIC BIOCHIP [23]

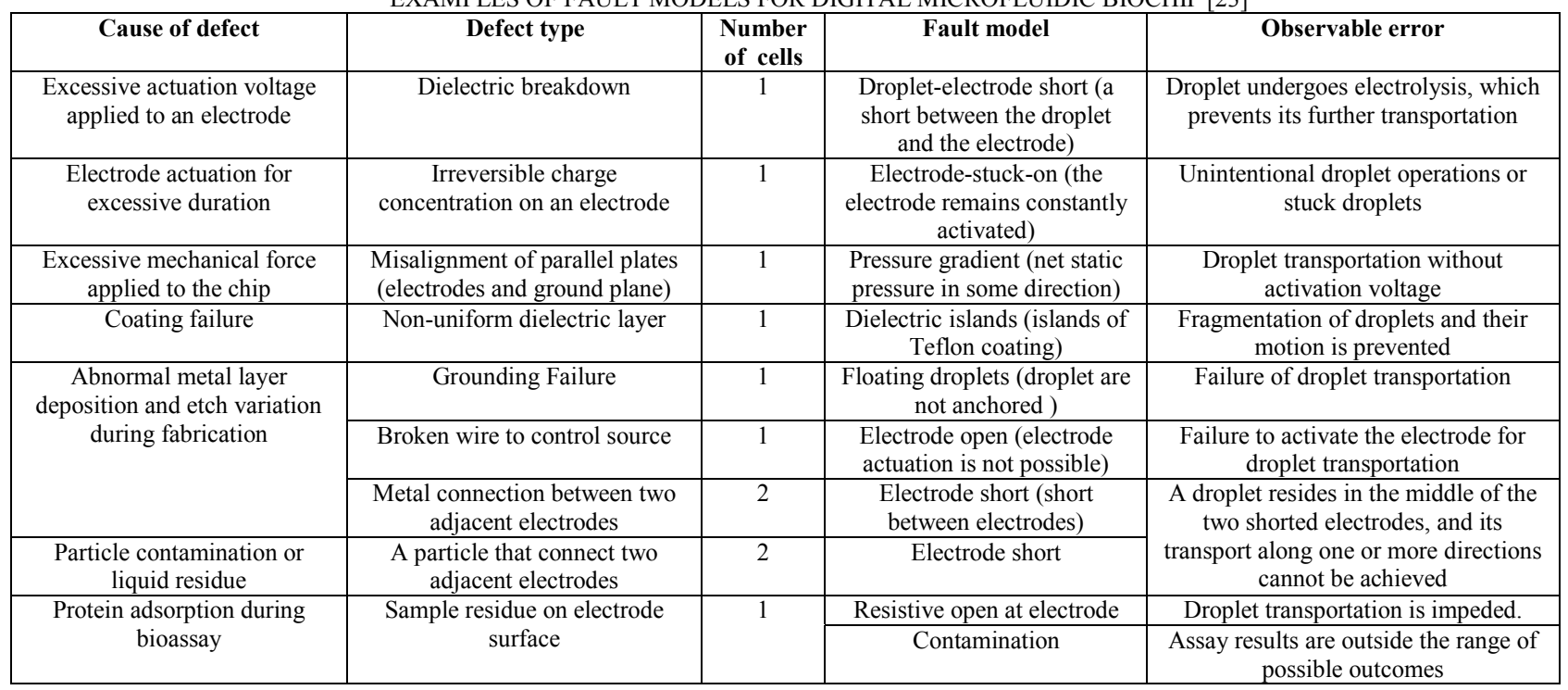

The number of control pins can be reduced by connecting together electrodes with mutually-compatible activation sequences, and addressing them using a single control pin. Therefore, the resulting electrode-access method is referred to as a broadcast addressing. The first step here is to partition the electrodes into groups. For all the electrodes in any group, the corresponding activation sequences must be pairwisecompatible. The problem of finding an optimal partition that leads to the minimum number of groups can be easily mapped to the problem of determining a minimal clique partition from graph theory. The minimum number of groups yields the minimum number of control pins.

\section{TESTING}

In this section, we describe recent advances in the testing of digital microfluidic biochips and fault localization techniques.

\section{A. Fault Modeling}

Faults in digital microfluidic systems can be classified as being either catastrophic or parametric. Catastrophic faults lead to a complete malfunction of the system, while parametric faults cause degradation in the system performance. Table I lists some common failure sources, defects and the corresponding fault models for catastrophic faults in digital microfluidic lab-on-chip.

\section{B. Structural Test Techniques}

A unified test methodology for digital microfluidic biochips has recently been presented, whereby faults can be detected by controlling and tracking droplet motion electrically [14]. Test stimuli droplets containing a conductive fluid (e.g., KCL solution) are dispensed from the droplet source. These droplets are guided through the unit cells following the test plan towards the droplet sink, which is connected to a capacitive detection circuit. Most catastrophic faults result in a complete cessation of droplet transportation. Therefore, we can determine the fault-free or faulty status of the system by simply observing the arrival of test stimuli droplets at selected ports. An efficient test plan ensures that testing does not conflict with the normal bioassay, and it guides test stimuli droplets to cover all the unit cells available for testing. The microfluidic array can be modeled as an undirected graph, and the pathway for the test droplet can be determined by solving the Hamiltonian path problem.

Even though most catastrophic faults lead to a complete cessation of droplet transportation, there exist differences between their corresponding erroneous behaviors. For instance, to test for the electrode-open fault, it is sufficient to move a test droplet from any adjacent cell to the faulty cell. The droplet will always be stuck during its motion due to the failure in charging the control electrode. On the other hand, if we move a test droplet across the faulty cells affected by an electrode-short fault, the test droplet may or may not be stuck depending on its flow direction. In [15], a solution based on Euler paths in graphs is described for detecting electrode shorts.

Despite its effectiveness for detecting electrode shorts, testing based on an Euler path suffers from long test application time. More recently, a cost-effective testing methodology referred to as "parallel scan-like test" has been proposed [24]. The method is named thus because it manipulates multiple test droplets in parallel to traverse the target microfluidic array, just as test stimuli can be applied in parallel to the different scan chains in an integrated circuit.

A drawback of the above "structural" test methods is that they focus only on physical defects, and they overlook module functionality. A defect-free microfluidic array can also malfunction in many ways. For example, a defect-free reservoir may result in large volume variations when droplets are dispensed from it. A splitter composed of three defect-free electrodes may split a big droplet into two droplets with significantly unbalanced volumes. These phenomena, referred 
to as malfunctions, are not the result of electrode defects. Instead, they are activated only for certain patterns of droplet movement or fluidic operations.

\section{Functional Testing Techniques}

Functional testing involves test procedures to check whether groups of cells can be used to perform certain operations, e.g., droplet mixing and splitting. For the test of a specific operation, the corresponding patterns of droplet movement are carried out on the target cluster of cells. If a target cell cluster fails the test, e.g., the mixing test, we label it as a malfunctioning cluster. As in the case of structural testing, fault models must be developed for functional testing. Malfunctions in fluidic operations are identified and included in the list of faults.

Functional test methods to detect the defects and malfunctions have recently been developed. In particular, dispensing test, mixing test, splitting test, and capacitive sensing test have been described in [23] to address the corresponding malfunctions.

\section{CONCLUSIONS}

We have presented a survey of research on design automation and test techniques for digital microfluidic biochips. We first provided an overview of the digital microfluidic platform, and then highlighted advances in synthesis and droplet routing techniques. Practical design techniques for achieving high throughout with a small number of control pins have been presented. Common defects have been identified and related to logical fault models. Based on these fault models, test techniques for emerging lab-on-chip devices and digital microfluidic modules have been presented.

\section{ACKNOWLEDGMENT}

The author thanks colleagues at Duke University and Advanced Liquid Logic, Inc., who have contributed to this work. They include Fei Su, Tao Xu, Yang Zhao, William Hwang, Phil Paik, Vamsee Pamula, and Richard Fair. This work was supported by the National Science Foundation under Grants IIS-0312352, CCF-0541055, and CCF-0914895, and the National Institute of General Medical Sciences of the National Institute of Health, grant \# R44GM072155.

\section{REFERENCES}

[1] A. Guiseppi-Elie et al., "Design of a subcutaneous implantable biochip for monitoring of glucose and lactate", IEEE Sensors Journal, vol. 5, pp. 345$355,2005$.

[2] R. B. Fair et al., "Chemical and biological applications of digitalmicrofluidic devices", IEEE Design \& Test of Computers, vol. 24, pp. 10$24,2007$.

[3] E. A. Ottesen et al., "Microfluidic digital PCR enables multigene analysis of individual environmental bacteria", Science, vol. 314, pp. 1464-1467, 2006.

[4] V. Srinivasan, V. K. Pamula, and R. B. Fair, "An integrated digital microfluidic lab-on-a-chip for clinical diagnostics on human physiological fluids", Lab on a Chip, vol. 4, pp. 310-315, 2004.

[5] E. Verpoorte and N. F. D. Rooij, "Microfluidics meets MEMS", Proceedings of IEEE, vol. 91, pp. 930-953, 2003.

[6] Semiconductor Industry Association, International Technology Roadmap for Semiconductors (ITRS), 2007. [Online]. Available: http://www.itrs.net/Links/2007ITRS/Home2007.htm
[7] A. S. Bedekar, et al., "System-level simulation of flow-induced dispersion in lab-on-a-chip systems", IEEE Trans. CAD, vol. 25, pp. 294-304, 2006.

[8] Y. Wang, Q. Lin, and T. Mukherjee, "Composable behavioral models and schematic-based simulation of electrokinetic lab-on-a-chip systems", IEEE Trans. CAD, vol. 25, pp. 258-273, 2006.

[9] M. G. Pollack, R. B. Fair, and A. D. Shenderov, "Electrowetting-based actuation of liquid droplets for microfluidic applications", Applied Physics Letters, vol. 77, pp. 1725-1726, 2000.

[10] S. K. Cho, H. J. Moon, and C. J. Kim, "Creating, transporting, cutting, and merging liquid droplets by electrowetting-based actuation for digital microfluidic circuits", Journal of Microelectromechanical Systems, vol. 12, pp. 70-80, Feb 2003.

[11] D. Chatterjee, B. Hetayothin, A. R. Wheeler, D. J. King, and R. L. Garrell, "Droplet-based microfluidics with nonaqueous solvents and solutions", Lab on a Chip, vol. 6, pp. 199-206, 2006.

[12] J. Berthier, Microdrops and Digital Microfluidics: Processing, Development, and Applications (Micro \& Nano Technologies), William Andrew Publishing, 2007.

[13] F. Su and K. Chakrabarty, "Architectural-level synthesis of digital microfluidics-based biochips", Proc. IEEE International Conference on CAD, pp. 223-228, 2004.

[14] F. Su, S. Ozev, and K. Chakrabarty, "Ensuring the operational health of droplet-based microelectrofluidic biosensor systems", IEEE Sensors, vol. 5, pp. 763-773, 2005.

[15] F. Su, W. Hwang, A. Mukherjee, and K. Chakrabarty, "Testing and diagnosis of realistic defects in digital microfluidic biochips", Journal of Electronic Testing: Theory and Applications, vol. 23, pp. 219-233, 2007.

[16] F. Su and K. Chakrabarty, "Unified high-level synthesis and module placement for defect-tolerant microfluidic biochips", Proc. IEEE/ACM Design Automation Conference, pp. 825-830, 2005.

[17] F. Su and K. Chakrabarty, "Design of fault-tolerant and dynamicallyreconfigurable microfluidic biochips", Proc. DATE Conf., pp. 1202-1207, 2005.

[18] F. Su and K. Chakrabarty, "Defect tolerance for gracefully-degradable microfluidics-based biochips", Proc. IEEE VLSI Test Symposium, pp. 321-326, 2005.

[19] F. Su, W. Hwang, and K. Chakrabarty, "Droplet routing in the synthesis of digital microfluidic biochips", Proc. Design, Automation and Test in Europe (DATE) Conference, pp. 323-328, 2006.

[20] F. Su, S. Ozev, and K. Chakrabarty, "Test planning and test resource optimization for droplet-based microfluidic systems", Journal of Electronic Testing: Theory and Applications, vol. 22, pp. 199-210, 2006.

[21] F. Su and K. Chakrabarty, "Module placement for fault-tolerant microfluidics-based biochips", ACM Transactions on Design Automation of Electronic Systems, vol. 11, pp. 682-710, 2006.

[22] T. Xu and K. Chakrabarty, "Droplet-trace-based array partitioning and a pin assignment algorithm for the automated design of digital microfluidic biochips", Proc. IEEE/ACM International Conference on Hardware/Software Codesign and System Synthesis, pp. 112-117, 2006.

[23] T. Xu and K. Chakrabarty, "Functional testing of digital microfluidic biochips", Proc. IEEE International Test Conference, 2007.

[24] T. Xu and K. Chakrabarty, "Parallel scan-like test and multiple-defect diagnosis for digital microfluidic biochips", IEEE Trans. Biomedical Circuits and Systems, vol. 1, pp. 148-158, June 2007.

[25] T. Xu and K. Chakrabarty, "Integrated droplet routing in the synthesis of microfluidic biochips", Proc. IEEE/ACM Design Automation Conference, pp. 948-953, 2007.

[26] T. Xu and K. Chakrabarty, "A cross-referencing-based droplet manipulation method for high-throughput and pin-constrained digital microfluidic arrays", Proc. DATE Conf., pp. 552-557, 2007.

[27] T. Xu and K. Chakrabarty, "Broadcast electrode-addressing for pinconstrained multi-functional digital microfluidic biochips", Proc. IEEE/ACM Design Automation Conference, pp. 173-178, 2008.

[28] P.-H. Yuh et al., "A progressive-ILP based routing algorithm for crossreferencing biochips", Proc. DAC, pp. 284-289, 2008.

[29] M. Cho and D. Z. Pan, "A high-performance droplet router for digital microfluidic biochips”, Proc. Int. Symp. Physical Design (ISPD), 2008.

[30] S.-K. Fan, C. Hashi, and C.-J. Kim, "Manipulation of multiple droplets on $\mathrm{N} \times \mathrm{M}$ grid by cross-reference EWOD driving scheme and pressurecontact packaging", Proc. MEMS, pp. 694-697, 2003. 